\title{
Justification for meso-scale modelling in quantifying constraint during creep crack growth.
}

\author{
K Nikbin \\ Department of Mechanical Engineering \\ Imperial College \\ London SW7 2BX \\ United Kingdom
}

Keywords: Creep crack growth, fracture mechanics, creep damage, constraint, failure strain, multiaxiality, $C^{*}$, $Q$ parameter

\begin{abstract}
.
Meso-scale models over a range of 50-500 $\mu \mathrm{m}$ are employed for predicting crack growth in the creep range. They make use of fracture mechanics, creep damage mechanics under multiaxial loading and limit analysis techniques. A study is made of the several levels of approximations and validations in the modelling process that are needed in order to make the method an acceptable tool for use in a safe defect assessment procedure. The NSW mesoscale model developed previously has been used to compare crack growth rate in a $316 \mathrm{H}$ stainless steel tested at $550{ }^{\circ} \mathrm{C}$ using Compact Tension (CT) and Centre Cracked Panel (CCP) specimens. In this way the steady crack growth region can be explained by an upper/lower bound cracking rate based on the level of multiaxial constraint. However the model only quantifies the upper/lower plane stress/strain bounds and does not quantify the intermediate levels of constraint. Therefore a two-parameter concept to predict constraint and the various steps is employed to improve the predictions for defect assessment in components. Preliminary results using two-dimensional modelling of a CCP specimen under plane strain conditions suggest that the method could be used to predict conservative estimates for crack growth rates when compared to experimental results. Further numerical analysis using threedimensional models of actual geometric sizes are necessary to confirm the usefulness of the two parameter method in defect assessment procedures.
\end{abstract}

\section{Introduction}

Mechanistic modelling at the micro level or atomistic levels will invariably be unable to describe the problem at the macro level without making numerous assumptions, simplifications and reductions in the number of variables employed. In high temperature 
fracture mechanics applications, modelling at the meso-scale level (a few grain sizes) [1-4] is used to explain the physical phenomenon and links the results to the macro-scale 'realistic' component size conditions. Modelling of crack growth predictive methods need further simplification before being implemented into defect assessment codes [5-7] in order to ensure sufficiently conservative, yet not over-conservative, estimates for remaining life. This task is made more difficult due to the fact that creep is highly stress sensitive in nature and therefore contains an inherent scatter in data. Creep damage and fracture mechanics models based on fundamental time-dependent damage or crack driving force concepts usually use short-term data (100-10000 hours) taken from small specimen testing. This is then used to predict longterm failures of components in service with initial design lives in the region of upto 200,000 hours. Verification and validation therefore becomes necessary in order to achieve this predictive extrapolation. The various steps necessary for these type of procedure to be implemented is continually being developed and improved.

In this paper the modelling for these procedures is presented using the NSW [1-3] mesofracture mechanics model based on the multiaxial failure strain concept for developing damage ahead of a crack tip. In this way the initiation stage and the steady crack growth region can be explained and bound by an upper/lower plane strain/stress line for cracking rate based on the level of multiaxial constraint. However the model does not quantify the intermediate levels of constraint. Therefore a two-parameter extension to predict constraint [8-9] and the various steps necessary to improve the predictions for defect assessment in components are considered. The paper assesses the links between the meso-scale modelling to macro scale crack growth.

\section{Model for Creep Crack Growth}

The arguments for correlating high temperature crack growth data essentially follow those of elastic-plastic fracture mechanics methods. For creeping situations [1-5] where elasticity dominates $K$ may be sufficient to predict crack growth. However as creep is a non-linear time dependent mechanism even in situation where small scale creep may exist linear elasticity my not be the answer. By using the $J$ definition to develop the fracture mechanics parameter $C^{*}$ it is possible to correlate time-dependent crack growth using non-linear fracture mechanics concepts. 
A simplified expression for stress dependence of creep is given by a power law equation which is often called the Norton's creep law and is comparable to the power law hardening material giving;

$$
\varepsilon=A^{\prime} \sigma^{N}
$$

and by analogy for a creeping material

$$
\dot{\varepsilon}=A \sigma^{n} \quad \text { or } \quad \frac{\dot{\varepsilon}}{\dot{\varepsilon}_{0}}=\left(\frac{\sigma}{\sigma_{0}}\right)^{n}
$$

and

$$
t_{r}=\frac{\varepsilon_{f}}{\dot{\varepsilon}_{0}}\left(\frac{\sigma}{\sigma_{0}}\right)^{v}
$$

where $A^{\prime}, A, N, n, \dot{\varepsilon}_{o}$, is $1 /$ hour and $\mathrm{C}, \sigma_{O}, n$ and $v$ are material constants and $\varepsilon_{f}$ represents the material uni-axial creep ductility at stress $\sigma_{0} . \varepsilon, \dot{\varepsilon}$ and $\sigma$ are the strain, creep strain rate and applied stress respectively. Equation (2) is used to characterise the steady state (secondary) creep stage where the hardening by dislocation interaction is balanced by recovery processes. The typical value for $n$ is between 5 and 12 for most metals. When $N=n$ for creep and plasticity it is assumed that the state of stress is characterised in the same manner for the two conditions. As an example for material data used in equations (2) and (3) Table (1) represents material data for a $316 \mathrm{H}$ type stainless steel. This data will be used in the numerical analysis and the modelling in the latter sections.

The stress fields characterised by $K$ in elasticity will be modified to the stress field characterised by the $J$ integral in plasticity in the region around the crack tip. In the case of large scale creep where stress and strain rate determine the crack tip field the $C^{*}$ parameter is analogous to $J$. The $C^{*}$ integral which can be characterized by the HRR filed [10] and can be experimentally derived [3,5] has been widely accepted as the fracture mechanics parameter for this purpose $[1-7,11]$. The crack growth in creep can be described in various ways using 
different correlating parameters [1-5]. However the parameter $C^{*}$ has been adopted as the appropriate parameter for mainly creep ductile conditions both in testing standards [2] and in defect assessment codes [6-7]. Immediately after loading, in the absence of plastic deformation the stress distribution ahead of a crack tip is given by the elastic stress intensity factor $K$. With time creep will cause stress redistribution until a steady state condition is reached which will be described by the creep fracture mechanics parameter $C^{*}$ [3]. The time taken for redistribution of $C^{*}$ to steady state $t_{T}$ is given by [3-5]

$$
t_{T}=\frac{K^{2}\left(1-v^{\prime 2}\right)}{E C^{*}(n+1)}
$$

where $K$ is the elastic stress intensity factor, $E$ is the Young's Modulus and $v^{\prime}$ is Poisson's ratio. The limits set in equation (4) can be practically utilised to identify the relative limits of the applicability of the parameter $C^{*}[5,12]$. It is found that creep crack growth rate $\dot{a}$ can be correlated satisfactorily in terms of $C^{*}$ by the relation $[11,12]$

$$
\dot{a}=D_{0} C^{* \phi}
$$

where $D_{0}$ and $\phi$ are material constants which can be measured experimentally or determined from a model of the cracking mechanism. The creep crack growth characteristics of materials can be determined experimentally (using test procedures specified in ASTM E1457-01) [4] or they can be predicted from uniaxial creep data and limit load analysis [4,6-7]. In both instances fracture mechanics concepts are employed and are subsequently simplified to a workable level in these codes.

Under steady state condition a crack growth model called the NSW [1-2] has been developed in which a process zone $r_{c}$ of the size of 10-100 microns (approximately in the order of the size of a few grains, is postulated at a crack tip. It is supposed that this zone of size $r_{C}$ encompasses the region over which creep damage accumulates locally at the crack tip. It is also assumed that an element of material first experiences damage when it enters the process zone at $r_{C}$ and that crack advance takes place when the creep ductility appropriate to the state of stress at the crack tip $\varepsilon_{f}^{*}$ is exhausted there. Figure 1 shows a schematic diagram of the model in which the creep zone is described by a series of pseudo uniaxial specimens which different stress distributions described by the HRR [10] stress/strain rate fields. With this 
approach, for a material with uni-axial creep properties given by equations (2) and (3) the constants in equation (5) are subsequently derived [1] as

$$
\phi=v(n+1)
$$

and

$$
D_{0}=\left[\frac{n+1}{n+1-v}\right] \frac{\dot{\varepsilon}}{\varepsilon_{f}^{*}}\left[\frac{1}{I_{n} \sigma_{0} \dot{\varepsilon}_{0}}\right]^{v /(n+1)} r_{c}^{(n+1-v) /(n+1)}
$$

and in most cases $v \cong n$ simplifying equation (7) further to

$$
D_{0}=n+1 \frac{\dot{\varepsilon}}{\varepsilon_{f}^{*}}\left[\frac{1}{I_{n} \sigma_{0} \dot{\varepsilon}_{0}}\right]^{n /(n+1)} r_{c}^{1 /(n+1)}
$$

where $I_{n}$ is a normalising factor, which depends on $n$ and the state of stress at the crack tip and uniaxial failure strain $\varepsilon_{f}$. For plane stress conditions the appropriate failure strain $\varepsilon_{f}^{*}$ is taken to be the uniaxial failure strain, $\varepsilon_{f}$, and for plane strain situations $\varepsilon_{f}^{*}$ can be as low as $\varepsilon_{f} / 30$ [11-12]. Experimentally it has been shown that the upperbound of 30 is conservative for most creep ductile situations.

Equation (8) is further simplified to a workable 'Engineering' model by making a number of assumptions of the variables employed. For most engineering alloys materials the value of $n>>1$ (usually between 6-12). Also it is seen that in equations (7) and (8) there is relatively insensitivity to the magnitude of $r_{c}$ which can therefore range between a few grain sizes (50500) microns. Furthermore, from examination of a wide range of experimental creep crack growth data it has been found that $D_{o}$ is most sensitive to creep uniaxial failure strain allowing equation (5) to be reasonably approximated for many materials by

$$
\dot{a}=\frac{3 C^{* \phi}}{\varepsilon_{f}^{*}}
$$

where $\dot{a}$ is in $\mathrm{mm} / \mathrm{h}, C^{*}$ is in $\mathrm{MJ} / \mathrm{m}^{2} \mathrm{~h}$ and $\varepsilon_{f}^{*}$ is the appropriate failure strain (as a fraction). The term $\phi \approx n /(n+1)$ and can also approximated to $\phi=0.85$ to cover a range of engineering materials [2,4]. This therefore is a clear example of how a detailed meso-scale model needs to be simplified to cover a wide range of conditions. 
These bounds derived experimentally can be verified using a stress state argument linked to models of multi-axial creep failure strain, resulting from void growth at the grain size level, proposed by Cocks and Ashby [13]. Under a triaxial stress state the void growth model proposed uses the ratio of the mean stress to the equivalent stress $\left(\sigma_{m} / \sigma_{e}\right)$ to estimate the appropriate crack-tip creep ductility $\varepsilon_{f}{ }^{*}$ in terms of the uniaxial ductility $\varepsilon_{f}$. The model gives this ratio as

$$
\frac{\varepsilon_{f}^{*}}{\varepsilon_{f}}=\sinh \left[\frac{2}{3}\left(\frac{n-0.5}{n+0.5}\right)\right] / \sinh \left[2\left(\frac{n-0.5}{n+0.5}\right) \frac{\sigma_{m}}{\sigma_{e}}\right]
$$

Clearly from equation (10) the ratio $\varepsilon_{f}^{*} / \varepsilon_{f}$ is sensitive to the relevant $\left(\sigma_{m} / \sigma_{e}\right)$ at the crack tip whose value in turn is sensitive to the creep index $n$ and the numerical techniques used to estimate them. As a typical example it has been shown, using numerical Finite Element elastic/plastic creep analysis [14], that for Compact Tension (CT) specimens, the mean values of $\sigma_{m} / \sigma_{e}$ for steady state has been calculated to be in the range 0.6 and 2.5, under plane stress $(P S)$ and plane strain $(P E)$ conditions respectively. If these values are replaced in equation (10) and using $n=12$ calculation for $\varepsilon_{f}^{*} P E$ and $\varepsilon_{f}^{*} P S$ gives

$$
\varepsilon_{f P E}^{*} \approx \frac{1}{60} \varepsilon_{f} \text { and } \varepsilon_{f P S}^{*} \approx \frac{1}{2} \varepsilon_{f}
$$

The range is shown in figure 2 which suggests a factor of 30 used in the NSW predictions is appropriate. The small differences that exist between the NSW model and the predictions from the FE analysis are likely to be due to the different values of $\varepsilon_{f}$ used.

Overall from data gathered on a range of materials and geometries it has been shown that the factor of 30 can be achieved but only in extreme circumstances [2,11]. However for specific materials and test conditions the range of crack growth, with respect to constraint, is likely to show a smaller range than the predicted factor of 30 [15].

\section{Comparison with experimental results}


In this section a specific test example is presented to link the experimental to the modelling and numerical analysis. This consists of tests carried out at $550{ }^{\circ} \mathrm{C}$ on a $316 \mathrm{H}$ type stainless steel using compact tension (CT) and centre crack panel (CCP) specimens. The relevant material properties of the steel, used in the analysis, are shown in Table 1.

No detail of test procedures will be reported in this paper as they have been published previously [15]. Essentially test procedures for testing the CT and CCP specimens follow the ASTM testing method for elevated temperatures [4]. Figure 3 shows the two geometries used in testing a $316 \mathrm{H}$ stainless steel at $550{ }^{\circ} \mathrm{C}$ and figure 4 shows the crack growth rate data for the specimens tested correlated versus $C^{*}$.

By using various sizes of CT and one size of CCP the effects of crack tip constraint and the resulting crack growth rates can be compared. Experimentally it is clear that for the test conditions described in figure 4 the larger the specimen the faster the cracking rate (within the level of scatter that exists in the data). Also the CCP geometry exhibits the lowest cracking rate since it is of lower constraint than the CT specimens.

For clarity the size and geometry effect is better highlighted in Figure 5 where the best mean value lines for the different geometries are shown. The predicted plane stress/strain bounds using equations $(5,7)$ and material properties from Table 1 , are also shown in the figure as well as the mean line of the whole data set. It is clear that a factor of 30 on plane strain is over conservative for these short term tests ( $<5000$ hours) of $316 \mathrm{H}$ stainless steel which exhibits a creep failure ductility $\varepsilon_{f}$ of about $20 \%$. This suggests insufficient constraint is achieved for these tests. The same may not be the case for long term tests where the reduction in crack tip plasticity and creep ductility may highlight geometric and size effects in terms of cracking rate.

As a design or a life assessment criterion, the choice of the upperbound plane strain line is bound to be too conservative for most practical situations. Therefore an improvement in the model is needed to be able to quantify the constraint level for different geometries and load levels. This may be achieved by a two parameter criterion described below. 


\section{Modelling High Temperature Constraint}

For steady state creep an analogy can be made between power law plasticity and power law creep as explained earlier in equation (1)-(3) under creep conditions, the hydrostatic $\sigma_{m}$ and the von Mises, $\sigma_{e}$ stress fields can be given by [8]:

$$
\begin{aligned}
& \frac{\sigma_{m}}{\sigma_{0}}=\left(\frac{C^{*}}{\dot{\varepsilon}_{0} \sigma_{0} I_{n} r}\right)^{1 /(n+1)} \tilde{\sigma}_{m}(\theta)+Q+\ldots \\
& \frac{\sigma_{e}}{\sigma_{0}}=\left(\frac{C^{*}}{\dot{\varepsilon}_{0} \sigma_{0} I_{n} r}\right)^{1 /(n+1)} \tilde{\sigma}_{e}(\theta)+\ldots
\end{aligned}
$$

The first term in equations (12) and (13) is the HRR field [13] and $Q$ is termed a 'constraint' factor which depends on the remote out of plane stresses. Steady state crack growth now depends on two parameters, $C^{*}$ and $Q$. Experimental and numerical investigations are therefore required to determine the importance of $Q$ in creep crack growth. From finite element studies the different levels of constraint due to specimen type and crack length have been quantified using the $Q$ parameters [9].

Two dimensional elastic-power law creep finite element analyses have been conducted to examine the effect of specimen size, type, geometry (different crack lengths) with the material properties (shown in Table 1) on the stress fields ahead of a crack. A hydrostatic $Q$ stress is used to quantify the influence of constraint observed and to provide a framework for assessing different specimen types. This approach is then applied to the creep crack growth analysis, using creep data derived from uniaxial tests.

From the FE analysis using ABAQUS [16] it is known that the constraint of a specimen will depend on its crack length, therefore, as the crack grows, the constraint will change. An expression for the crack growth rate based on the $Q$ stress $[8,9]$ by manipulating equations $(8,12-13)$ to give

$$
\dot{a}=\dot{\varepsilon}_{0} \frac{\varepsilon_{f}}{\varepsilon_{f}^{*}}\left(\frac{C^{*}}{I_{n} \sigma_{0} \dot{\varepsilon}_{0}}\right)^{n /(n+1)}(n+1) r_{c}^{1 /(n+1)} g
$$




$$
\text { where } g=1+\frac{3}{4} \frac{Q}{\sigma_{e}}\left(\frac{C^{*}}{I_{n} \sigma_{0} \dot{\varepsilon}_{0}}\right)^{-1 /(n+1)}(n+1) r_{c}^{1 /(n+1)} \text {, }
$$

and $\varepsilon_{f}^{*}$ is the multiaxial ductility evaluated at the appropriate $\sigma_{m} / \sigma_{e}$. It can be seen that as the constraint decreases the crack growth rate decreases as well. When $Q=0$, equation (14) reduces to the crack growth rate NSW prediction given by equation (8)

This has been investigated for a CCP using the conditions of the test performed on $316 \mathrm{H}$ steel at $550{ }^{\circ} \mathrm{C}$ where the crack grew from $a / W=0.34$ to $a / W=0.49$. The finite element predictions are based on equation (14) where $\dot{a}$ and $Q$ have been evaluated at the experimental value of $C^{*}$. The results are shown in Fig. 6 for steady state crack growth rates conditions.

The predicted crack growth rate, is comparable to the experimental data shown and is bound by the upper/lower plane strain/stress bound of equation (6) using the approximate NSW model. The model correctly predicts the trends seen in the experimental data and is shown to be conservative by a factor of approximately 3-5. Overall the predicative trends seem to compare well with the experimental data however further work is needed in order to reduce the conservatism by considering the effects sensitivity to mesh size and the effects of material properties used as input data.

\section{Conclusions}

Creep crack development in structures can be separated into two regimes of failure, consisting of initiation and a steady cracking behaviour both of which are affected by constraint. The paper deals with the steady state cracking regime. The paper presents an example of how the use of creep meso-scale fracture mechanics modelling techniques can be translated into an engineering assessment of crack growth rate. The various steps necessary for rationalization and simplifications of the models are highlighted and the the possible effects of material properties variability to crack growth predictions are presented. The parameter described as $C^{*}$ has been shown to correlate the steady state crack growth rate quite well. The meso-scale NSW model, which considers the development of a creep zone under a multi-axial stress state, has been used to produce a material independent engineering diagram bounding over the plane stress/strain steady state using the a multi-axial model of 
void growth to predict a multiaxial failure ductility. The NSW model, is used to describe crack growth behaviour in a range of engineering alloys, showing that the model does not have to perform under strict tolerances. From the model the most conservative bound chosen for defect assessment predictions would be the plane strain which gives a factor of 30 in crack growth rates in the extreme over plane stress conditions. However the range of crack growth due to constraint effects are not quantified. A two-parameter approach could be used to improve correlations for the cracking behaviour and reduce undue conservatism. The development of this parameter in both two dimensional and three dimensional models is computer intensive in terms of analysis and derivation. Preliminary results on a CCP laboratory geomety compared to actual tests carried out on a type $316 \mathrm{H}$ stainless steel at 550 ${ }^{\circ} \mathrm{C}$ show that the two-parameter method can be used to make conservative crack growth rate predictions. The results suggest that a comprehensive numerical analysis should be undertaken to compute $Q$ for three dimensional meshes in order to estimate its sensitivity to creep crack growth correlations in the range of plane stress/strain regime.

Acknowledgement: The author would like to thank Dr A. Bettinson for the numerical analysis and testing carried out in respect of this paper and the British Energy Generations Ltd for funding the work.

\section{References}

[1] Nikbin K.M., Smith D.J. and Webster G.A., "Prediction of Creep Crack Growth from Uniaxial Creep Data”, Proc. R. Soc. Lond., A 396, pp 183-197, 1984.

[2] Nikbin, K.M., Smith, D.J. and Webster, G.A., J Eng. Mat. and Tech.,(1986), ASME, 108, 186-191.

[3] Webster, G.A. \& Ainsworth, R.A., 'High Temperature Component Life Assessment', Chapman and Hall, London, 1994.

[4] Saxena, A. "Crack Growth under Non Steady-state Conditions", in 17th ASTM National Symposium on Fracture Mechanics. 1984. Albany, NY.

[5] ASTM E 1457-01, (2001), Standard Test Method for Measurement of Creep Crack Growth Rates in Metals, ASTM Standards 03.01.

[6] Ainsworth, R.A, editor R5:' Assessment Procedure for The High Temperature Response of Structures’, British Energy Generation Ltd. Procedure R5 Issue 2, 1999.

[7] British Standards- 7910: 1999, Guide to methods of assessing the acceptability of flaws in fusion welded structures, BSI, London, 1999. 
[8] Budden P.J., and Ainsworth R.A., "The Effect of Constraint on Creep Fracture Assessments", Int. Jnl. Fracture, 97, pp 237-247, 1999.

[9] Bettinson, A.D., O’Dowd N. P., Nikbin, K. M. and Webster, G. A., ' 'Two-parameter characterization of crack-tip fields under creep conditions’, IUTAM Conf., Japan, Apr. 2000.

[10] Rice J.R., and Rosengren G.F., "Plane Strain Deformation near a Crack Tip in a PowerLaw Hardening Material”, J. Mech. Phys. Solids, 16, pp. 1-12.

[11] Tan, M., Celard, N.J.C., Nikbin, K. , Webster, G.A., 'Comparison of creep crack initiation and growth in four steels tested in HIDA', Int. J. PVP, pp 2001, 737 - 747

[12] Kwon, O., Nikbin, K.M., Webster, G.A., JATA, K.V., 'Crack growth in the presence of limited creep deformation', Engineering Fracture Mechanics, 62, 1, 33-46, 1999.

[13] Cocks A.C.F. and Ashby M.F., "Intergranular Fracture in Power Law Creep under Multiaxial Stress", Metal Science, 14, pp395-402, 1980.

[14] Yatomi, M., Nikbin, K. M., O’Dowd, N. P., 'Creep crack growth prediction using a creep damage based approach', VAMAS TWA25 special publication, Int. J. PVP, 2003 (to be published)'

[15] Bettinson, A. D., O’Dowd, N. P, Nikbin, K., Webster, G. A.' Experimental investigation of constraint effects on creep crack growth', Proceedings of PVP'02, 2002 ASME pressure vessels \& piping (PVP) Conf., August 4-8, 2002, Vancouver, BC, Canada.

[16] ABAQUS v5.8, Hibbitt, Karlsson and Sorensen Inc., Providence, RI, 1998 
Table 1: Material properties of type $316 \mathrm{H}$ stainless steel at $550{ }^{\circ} \mathrm{C}$

\begin{tabular}{|c|c|c|c|c|c|c|c|}
\hline$E(\mathrm{GPa})$ & $\sigma_{Y}(\mathrm{MPa})$ & $\sigma_{\text {UTS }}(\mathrm{MPa})$ & $A$ & $n$ & $\dot{\varepsilon}_{\min } \mathrm{h}^{-1}$ & $\dot{\varepsilon}_{\mathrm{A}} \mathrm{h}^{-1}$ & $\varepsilon_{f} \%$ \\
\hline 130 & 190 & 595 & $1.56 \times 10^{-35}$ & 12 & $1.65 \times 10^{-5}$ & 18.3 & 21 \\
\hline
\end{tabular}




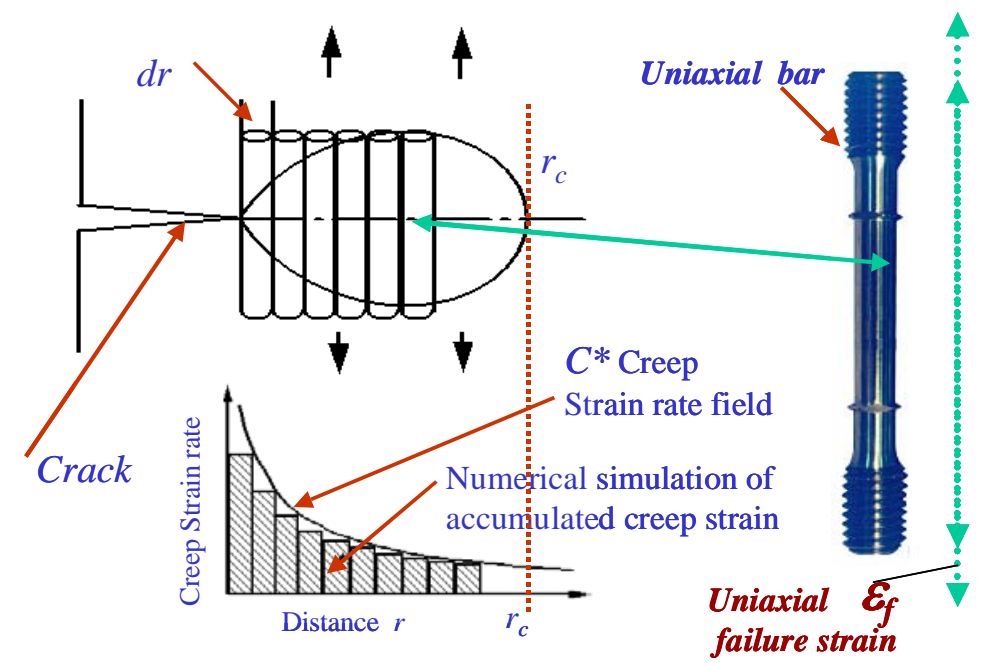

Figure 1: Schematic view of the creep process zone model in which the $C^{*}$ field dominates where units of length increments within the zone are compared to a uniaxial circular bar specimen used in actual creep tests.

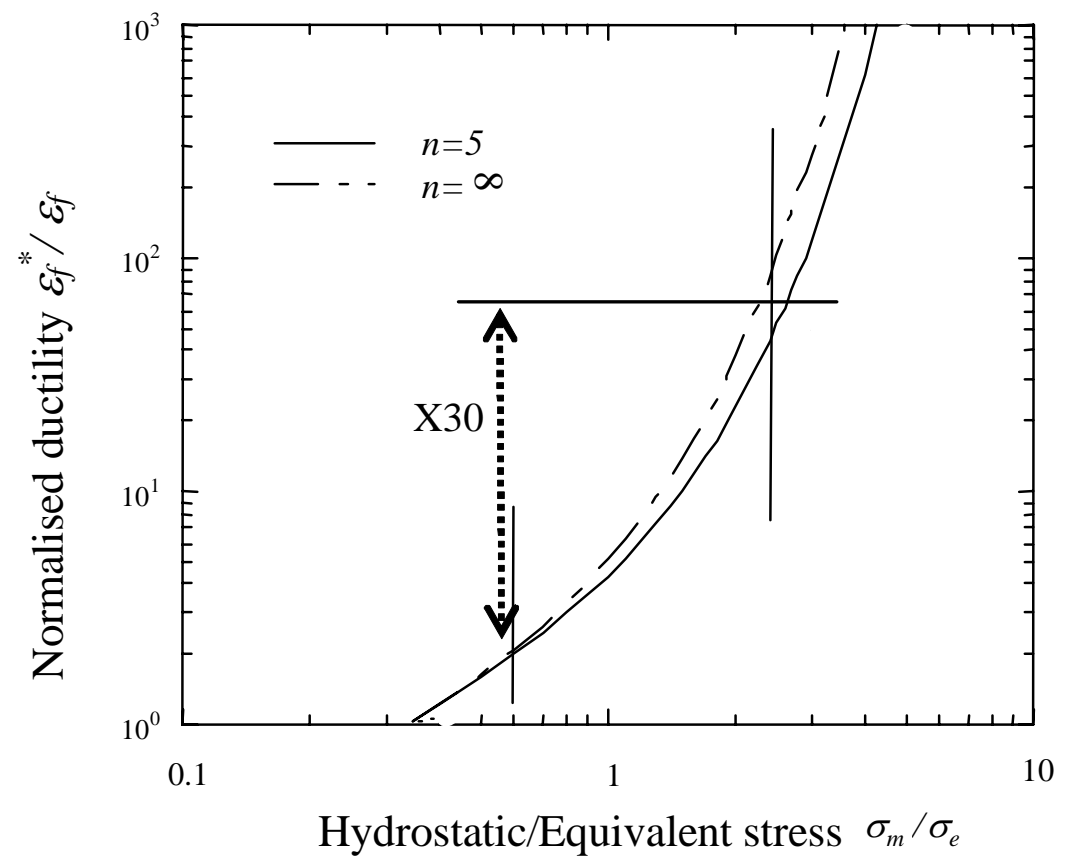

Figure 2: Relationship between appropriate crack-tip creep ductility $\varepsilon_{f}{ }^{*}$ and $\left(\sigma_{m} / \sigma_{e}\right)$ under a multi-axial stress state using equation (8) showing the range under plane stress and plane strain conditions for a CT specimen. 


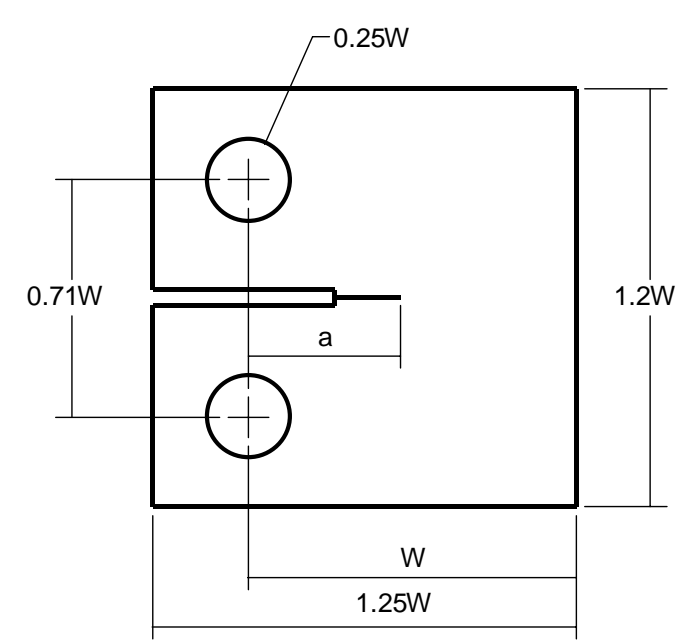

a)

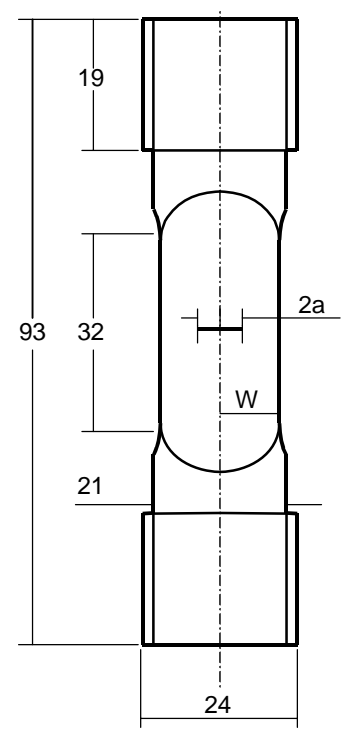

b)

Figure 3: Dimensions for CT, CCP specimens (in mm). Thicknesses $B=W / 2$ and $B=8 \mathrm{~mm}$ were used for the CT and CCP respectively

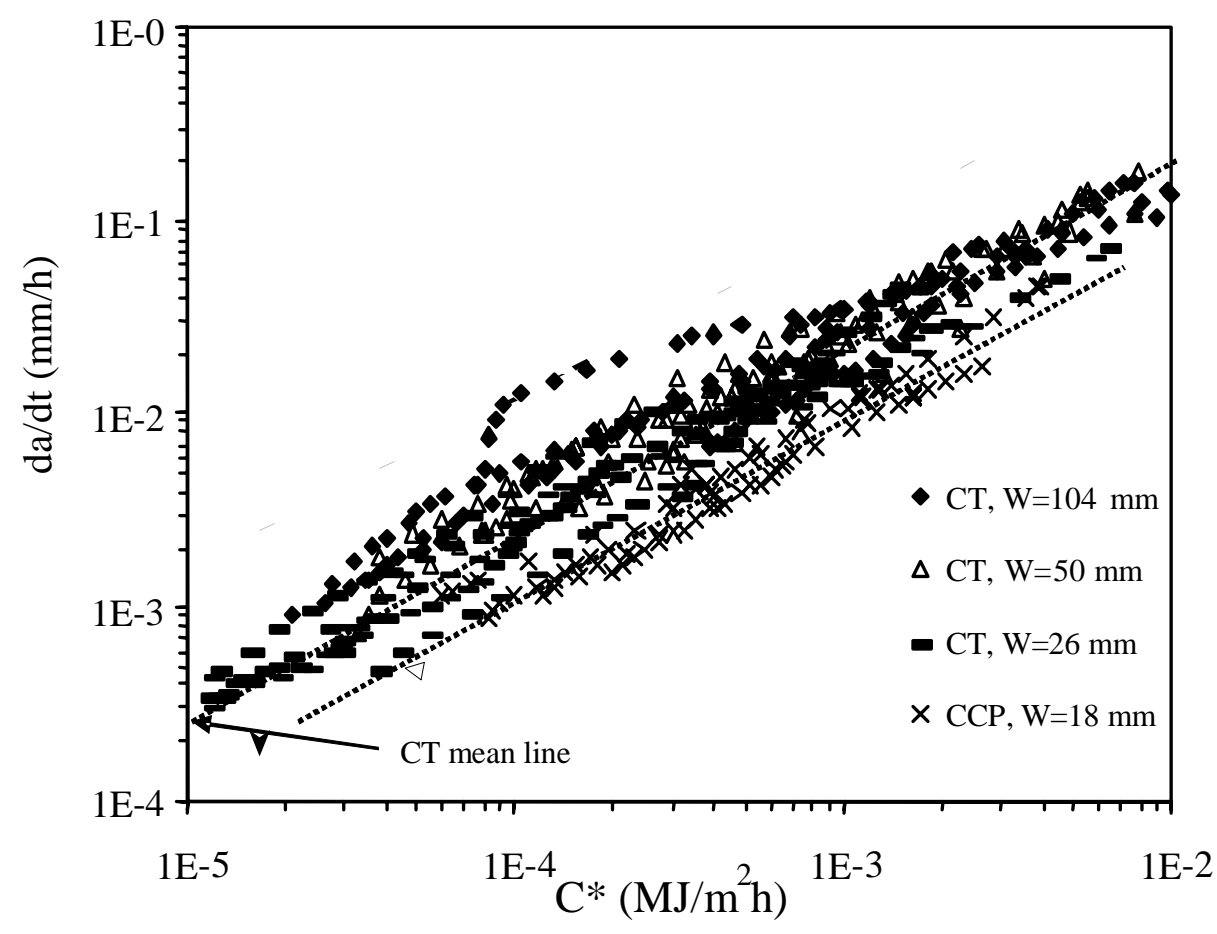

Figure 4: Crack growth rate versus $C^{*}$ for $316 \mathrm{H}$ type stainless steel tested at $550{ }^{\circ} \mathrm{C}$ [14] using different sizes of CT specimens and CCP specimens. 


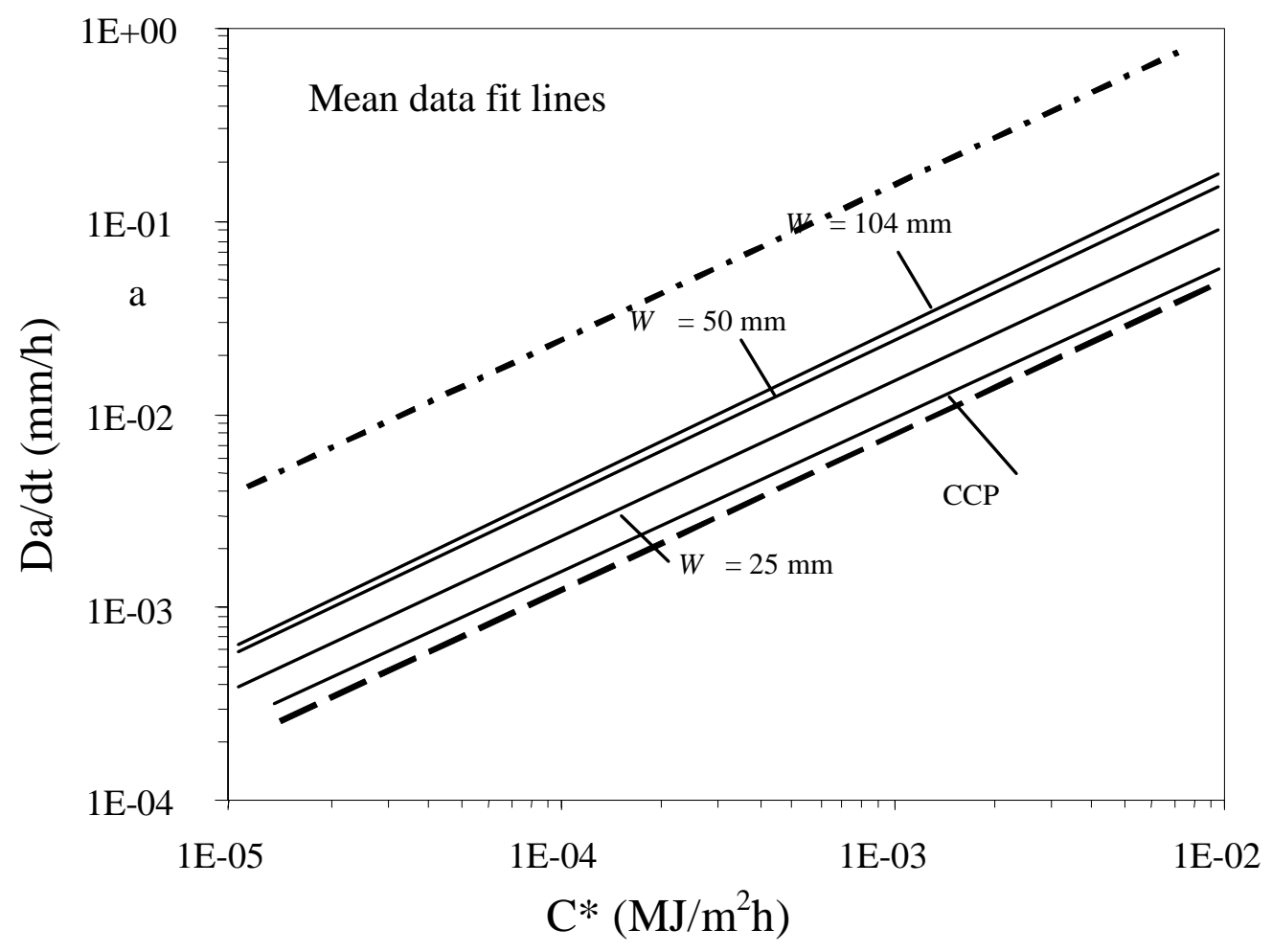

Figure 5: The best fit mean lines from Figure 4 showing the differences in cracking rate due to size and geometry. For $316 \mathrm{H}$ type stainless steel tested at $550{ }^{\circ} \mathrm{C}$.

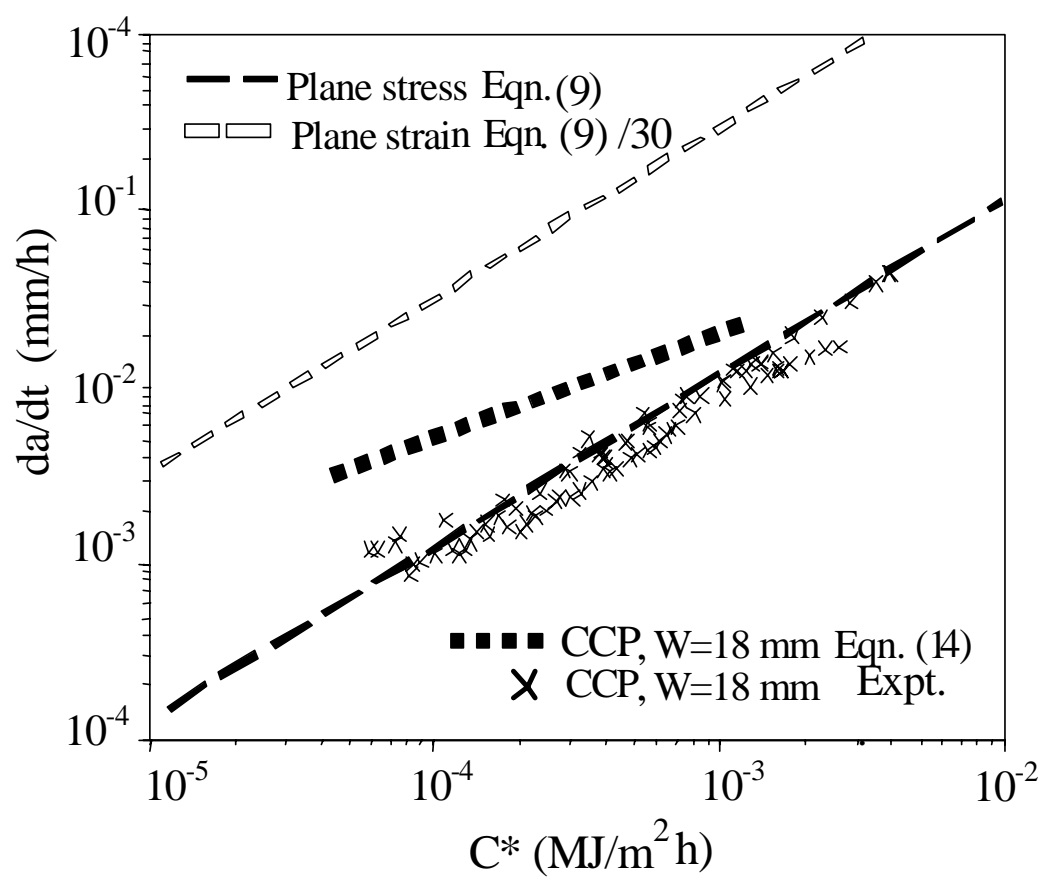

Fig 6: predicted and experimental crack growth rates for CCP $316 \mathrm{H}$ stainless steel tested at $550{ }^{\circ} \mathrm{C}$ using equations 9 (under plane stress using $\varepsilon_{f}$ and strain using $\varepsilon_{f} / 30$ ) and 14 
